\title{
The cost-effectiveness of pharmacist-physician collaborative care models vs usual care on time in target systolic blood pressure range in patients with hypertension: a payer perspective
}

\author{
Jessica S Jay, PharmD; Stephen C Ijioma, PharmD; David A Holdford, RPh, MS, PhD; Dave L Dixon, PharmD; \\ Evan M Sisson, PharmD; and Julie A Patterson, PharmD, PhD
}

\section{What is already known about this subject}

- Pharmacist-physician collaborative care models (PPCCMs) are effective at improving blood pressure (BP) control in patients with hypertension, including time in target range (TTR) for systolic BP.

- Increased TTR for systolic BP is associated with reduced risk of adverse cardiovascular events.

- PPCCM for hypertension management is likely cost-effective from the societal perspective when time-based costing methods are used for provider time and BP is studied as a continuous outcome.

\section{ABSTRACT}

BACKGROUND: Hypertension is highly prevalent in the United States, affecting nearly half of all adults (43\%). Studies have shown that pharmacist-physician collaborative care models (PPCCMs) for hypertension management significantly improve blood pressure (BP) control rates and provide consistent control of BP. Time in target range (TTR) for systolic BP is a novel measure of BP control consistency that is independently associated with decreased cardiovascular risk. There is no evidence that observed improvement in TTR for systolic BP with a PPCCM is cost-effective.

\section{What this study adds}

- This study assessed the costeffectiveness of PPCCM from the payer perspective while incorporating the increasingly utilized concept of TTR for systolic BP as a measure of BP control.

- For every 10,000 hypertension patients managed with PPCCM vs usual care over a 3-year time horizon, approximately 27 cardiovascular disease deaths, 29 strokes, 21 nonfatal myocardial infarctions, and 12 incident heart failure diagnoses are expected to be averted.

- Over a 3-year time horizon, PPCCM is expected to not only be less costly to administer but also result in downstream health care savings.

OBJECTIVE: To compare the cost-effectiveness of a PPCCM with usual care for the management of hypertension from the payer perspective.

METHODS: We used a decision analytic model with a 3-year time horizon based on published literature and publicly available data. The population consisted of adult patients who had a previous diagnosis of high BP (defined as office-based BP $\geq 140 / 90$ $\mathrm{mmHg}$ ) or were receiving antihypertensive medications. Effectiveness data were drawn from 2 published studies evaluating the effect of PPCCMs (vs usual care) on TTR for systolic BP and the impact of TTR for systolic BP on 4 cardiovascular outcomes (nonfatal

\begin{abstract}
Author affiliations
Jessica S Jay, PharmD; David A Holdford, RPh, MS, PhD; Dave L Dixon, PharmD; Evan M Sisson, PharmD; Stephen C Ijioma, PharmD; and Julie A Patterson, PharmD, PhD, Center for Pharmacy Practice Innovation, Virginia Commonwealth University School of Pharmacy, Richmond.
\end{abstract}

AUTHOR CORRESPONDENCE: Julie A Patterson, 804.828.0393; japatterson2@vcu.edu

J Manag Care Spec Pharm 2021;27(12):1680-90

Copyright $(2021$, Academy of Managed Care Pharmacy. All rights reserved.

myocardial infarction [MI], stroke, heart failure [HF], and cardiovascular disease [CVD] death). The model incorporated direct medical costs, including both programmatic costs (ie, direct costs for provider time) and downstream health care utilization associated with acute cardiovascular events. One-way sensitivity and threshold analyses examined model robustness.

RESULTS: In base-case analyses, PPCCM hypertension management was associated with lower downstream medical expenditures (difference: -\$162.86) and lower total program costs (difference: -\$108.00) when compared with usual care. PPCCM was associated with lower downstream medical 
expenditures across all parameter ranges tested in the deterministic sensitivity analysis. For every 10,000 hypertension patients managed with PPCCM vs usual care over a 3-year time horizon, approximately 27 CVD deaths, 29 strokes, 21 nonfatal MIs, and 12 incident HF diagnoses are expected to be averted.

CONCLUSIONS: This is the first study to evaluate the cost-effectiveness of PPCCM compared to usual care on TTR for systolic BP in adults with hypertension. PPCCM was less costly to administer and resulted in downstream health care savings and fewer acute cardiovascular events relative to usual care. Although further research is needed to evaluate the long-term costs and outcomes of PPCCM, payer coverage of PPCCM services may prevent future health care costs and improve patient cardiovascular outcomes.

Hypertension is highly prevalent in the United States, affecting nearly half of all adults (43\%). ${ }^{1}$ Hypertension is defined as having a systolic blood pressure (BP) $\geq 130$ or diastolic $\mathrm{BP} \geq 80 \mathrm{mmHg}$ and is a major risk factor for ischemic heart disease, heart failure (HF), stroke, chronic kidney disease, and death., ${ }^{1,2}$ Only about a quarter (24\%) of adults have their hypertension under control. From 2003-2014, it was estimated that hypertension accounted for \$131 billion per year in US health care costs. ${ }^{3}$

It has been shown that high BP variability is associated with increased risks of all-cause mortality, coronary heart disease, stroke, and end-stage renal disease. ${ }^{4-6}$ The concept of time in target range (TTR) for systolic BP is a novel measure of BP variability. ${ }^{7}$ A longitudinal study from 15 Veterans Affairs medical centers categorized TTR for systolic BP into 4 quartiles (0\%-25\%, 26\%-50\%, 51\%-75\%, and $76 \%-100 \%)$ and found an inverse and gradual association between TTR and all-cause mortality. ${ }^{7}$ To determine if TTR for systolic BP had an effect on cardiovascular outcomes, Fatani et al conducted a post hoc analysis of SPRINT (Systolic Blood Pressure Intervention Trial) trial data. ${ }^{8,9}$ In the fully adjusted models, the authors found that for every 1 standard deviation increase in TTR for systolic BP, the risk of a first major adverse cardiovascular event significantly decreased. $^{8}$ This study is consistent with other studies suggesting that greater variability in BP is associated with coronary heart disease, stroke, cardiovascular mortality, and all-cause mortality. ${ }^{4-6}$

Studies have shown that pharmacists play a key role within primary care settings in managing chronic diseases such as hypertension, and clinical pharmacy services decrease overall health care costs. ${ }^{10-12}$ A pharmacist-physician collaborative care model (PPCCM) is a practice model where pharmacists provide medication management for common primary care conditions, often under a collaborative practice agreement with a physician to adjust medications, and order necessary laboratory tests to monitor drug therapy. ${ }^{13}$ PPCCMs have been shown to not only be successful within an office-based setting, ${ }^{13}$ but even within barbershops and churches. ${ }^{14,15}$ A study by Matzke et al found significant improvements $(P<0.01)$ in hemoglobin, $\mathrm{BP}$, and cholesterol in patients with multiple chronic conditions who were in the PPCCM group compared to those seen by usual care. Additionally, hospitalizations declined within the PPCCM group, which led to an estimated cost savings of $\$ 2,619$ per patient. ${ }^{16}$ Carter et al have conducted multiple randomized clinical trials to assess the effectiveness of PPCCMs for hypertension management and found that patients treated under a PPCCM achieve significantly better mean BP and overall BP control rates. ${ }^{17,18}$ Recently, a study conducted by Dixon et al investigated the impact of PPCCM on TTR for systolic BP, as defined by the proportion of clinical encounters with systolic BP between 120 and $140 \mathrm{mmHg}$ during a 12-month follow-up period. ${ }^{13}$ The mean TTR for systolic BP was significantly higher among PPCCM patients $(46.2 \% \pm 24.3 \%)$ than patients who received usual care $(24.8 \% \pm 27.4 \%)(\mathrm{P}<0.0001) .{ }^{13}$ Additionally, a majority of patients in the usual care group had a TTR for systolic $\mathrm{BP}$ in the lowest quartile (0\%-25\%), while PPCCM patients were more likely to have TTR for systolic BP in the highest quartile (76\%-100\%). ${ }^{13}$

Despite the available evidence supporting PPCCM as an effective model at improving TTR for systolic BP compared to usual care ${ }^{13}$ and that patients with higher TTR for systolic BP have decreased risk of adverse cardiovascular events, ${ }^{7-9}$ no pharmacoeconomic analysis has combined these findings to model the cost-effectiveness of PPCCM. Therefore, we aimed to compare the cost-effectiveness of PPCCM with usual care on TTR for systolic BP in patients with hypertension. This study was conducted from the payer perspective to quantify the value added to a payer of covering PPCCM services.

\section{Methods}

\section{MODEL OVERVIEW}

This study used a decision analysis model (Figure 1) to evaluate the cost-effectiveness of 2 hypertension management practices, PPCCM and usual care. The population studied in this analysis consisted of adult patients who were previously diagnosed with hypertension (defined as office-based $\mathrm{BP} \geq 140 / 90 \mathrm{mmHg}$ ) or were receiving antihypertensive medications. ${ }^{13}$ A 3-year time horizon was chosen, reflecting the time frame of available data linking TTR for systolic 


\section{FIGURE 1 Decision Tree Analysis for the Cost-Benefit of PPCCM Compared With Standard Usual Care on TTR for Systolic Blood Pressure in Hypertension Management}

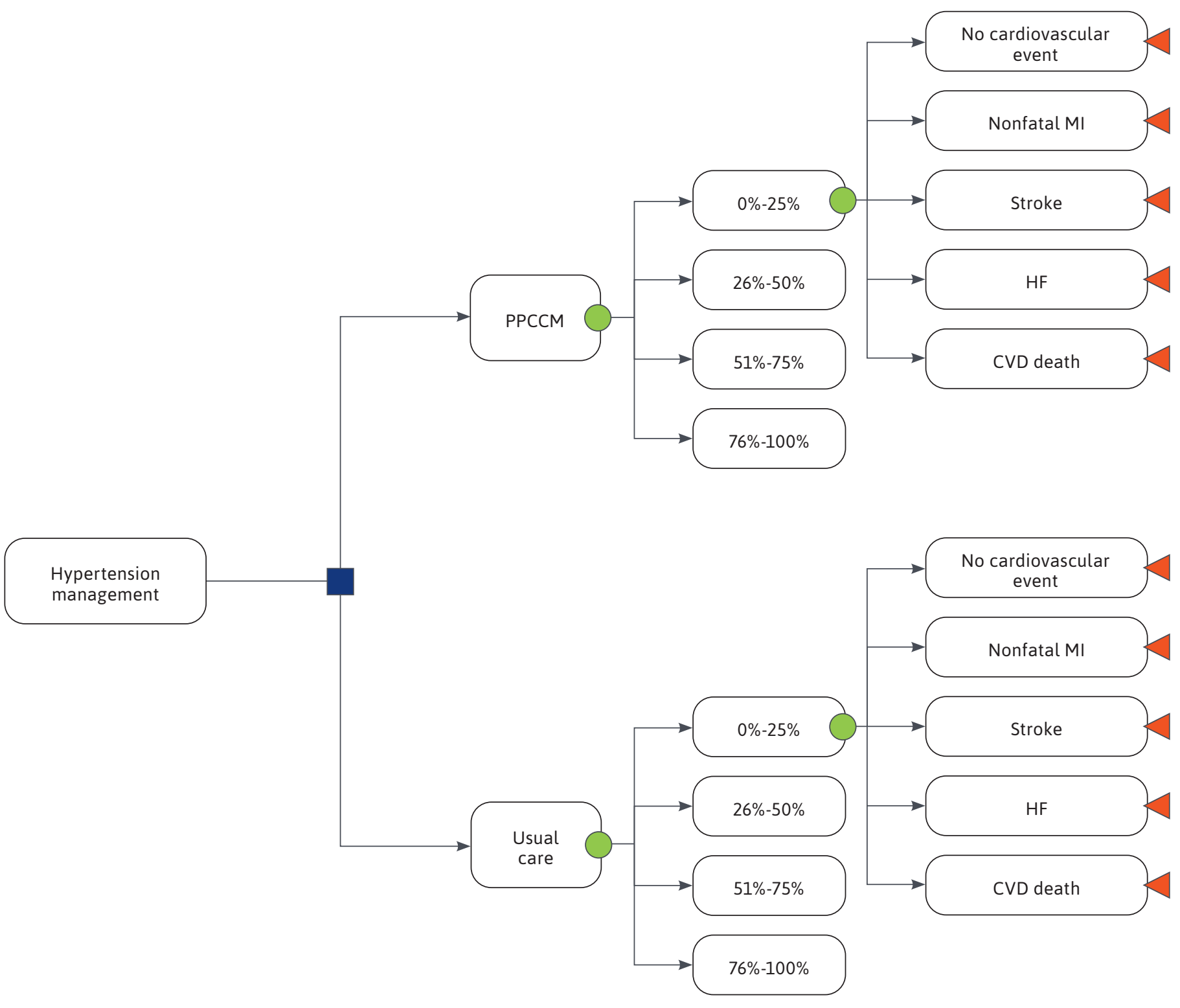

$C V D=$ cardiovascular disease $; \mathrm{HF}=$ heart failure; $M I=$ myocardial infarction; $P P C C M=$ pharmacist-physician collaborative care model; TTR=time in target range

BP (0\%-25\%, 26\%-50\%, 51\%-75\%, and 76\%-100\%) to cardiovascular outcome measures (nonfatal myocardial infarction [MI], stroke, HF, and cardiovascular disease [CVD] death). ${ }^{8,9}$ The time horizon is consistent with the follow-up duration from the SPRINT trial, which was terminated early given the clinical benefit of intensive BP control within 3 years of treatment., ${ }^{9,19}$ Further, the time horizon aligns with the shorter time frame utilized in cost-effectiveness models from the payer perspective. ${ }^{20}$ The model was developed in TreeAge Pro (TreeAge Software Inc). Institutional review board approval was not required as this research did not qualify as human subject research. 


\section{TABLE 1 Effectiveness and Cost Inputs}

\begin{tabular}{|c|c|c|c|}
\hline Variables & $\begin{array}{c}\text { Base-case } \\
\text { value }\end{array}$ & Range & Reference \\
\hline \multicolumn{4}{|c|}{ Probability of TTR for systolic BP by hypertension management approach } \\
\hline \multicolumn{4}{|l|}{ PPCCM } \\
\hline $0 \%-25 \%$ & 0.210 & $0.170-0.260$ & Dixon et al, $2020^{13}$ \\
\hline $26 \%-50 \%$ & 0.360 & $0.290-0.430$ & Dixon et al, $2020^{13}$ \\
\hline $51 \%-75 \%$ & 0.310 & $0.240-0.370$ & Dixon et al, $2020^{13}$ \\
\hline $76 \%-100 \%$ & 0.120 & $0.098-0.150$ & Dixon et al, $2020^{13}$ \\
\hline \multicolumn{4}{|l|}{ Usual care } \\
\hline $0 \%-25 \%$ & 0.550 & $0.400-0.600$ & Dixon et al, $2020^{13}$ \\
\hline $26 \%-50 \%$ & 0.340 & $0.270-0.400$ & Dixon et al, $2020^{13}$ \\
\hline $51 \%-75 \%$ & 0.050 & $0.042-0.064$ & Dixon et al, $2020^{13}$ \\
\hline $76 \%-100 \%$ & 0.060 & $0.044-0.066$ & Dixon et al, $2020^{13}$ \\
\hline \multicolumn{4}{|c|}{ Probability of cardiovascular events by TTR for systolic BP } \\
\hline \multicolumn{4}{|c|}{ Outcome event rates of patients in TTR for systolic BP $0 \%-25 \%$} \\
\hline Nonfatal MI & 0.035 & $0.027-0.045$ & Wright et al, $2015^{9}$ \\
\hline Stroke & 0.020 & $0.014-0.028$ & Wright et al, $2015^{9}$ \\
\hline Heart failure & 0.022 & $0.016-0.031$ & Wright et al, $2015^{9}$ \\
\hline CVD death & 0.017 & $0.012-0.024$ & Wright et al, $2015^{9}$ \\
\hline No cardiovascular event & 0.906 & - & Calculation \\
\hline \multicolumn{4}{|c|}{ Hazard ratio of patients in TTR for systolic BP $26 \%-50 \%$} \\
\hline Nonfatal MI & 0.83 & $0.57-1.18$ & Fatani et al, $2021^{8}$ \\
\hline Stroke & 0.83 & $0.55-1.27$ & Fatani et al, $2021^{8}$ \\
\hline Heart failure & 1.30 & $0.94-2.01$ & Fatani et al, $2021^{8}$ \\
\hline CVD death & 0.69 & $0.42-1.15$ & Fatani et al, $2021^{8}$ \\
\hline No cardiovascular event & 1.03 & - & Calculation \\
\hline \multicolumn{4}{|c|}{ Hazard ratio of patients in TTR for systolic BP $51 \%-75 \%$} \\
\hline Nonfatal MI & 0.87 & $0.61-1.24$ & Fatani et al, $2021^{8}$ \\
\hline Stroke & 0.58 & $0.36-0.93$ & Fatani et al, $2021^{8}$ \\
\hline Heart failure & 0.84 & $0.54-1.29$ & Fatani et al, $2021^{8}$ \\
\hline CVD death & 0.53 & $0.30-0.92$ & Fatani et al, $2021^{8}$ \\
\hline No cardiovascular event & 1.12 & - & Calculation \\
\hline \multicolumn{4}{|c|}{ Hazard ratio of patients in TTR for systolic BP $76 \%-100 \%$} \\
\hline Nonfatal MI & 0.69 & $0.46-1.04$ & Fatani et al, $2021^{8}$ \\
\hline Stroke & 0.40 & $0.22-0.73$ & Fatani et al, $2021^{8}$ \\
\hline Heart failure & 0.59 & $0.34-1.02$ & Fatani et al, $2021^{8}$ \\
\hline CVD death & 0.45 & $0.23-0.86$ & Fatani et al, $2021^{8}$ \\
\hline No cardiovascular event & 1.25 & - & Calculation \\
\hline
\end{tabular}

\section{EFFECTIVENESS: TIME IN TARGET SYSTOLIC BP RANGE AND CARDIOVASCULAR EVENT OUTCOMES}

Base-case parameters are listed in Table 1. The probabilities that patients managed with PPCCM and usual care would achieve levels of BP control within each of the 4 TTRs for systolic BP quartiles were based on previously published data. ${ }^{13}$ Although published data on the effectiveness of PPCCM had a 1-year study duration,,$^{13}$ subjects in the model were assumed to stay in the same quartile of target $\mathrm{BP}$ range over the 3-year time horizon to facilitate linking the PPCCM effectiveness data to the clinical data on the association between TTR for systolic BP and cardiovascular events.

Four cardiovascular events (nonfatal MI, stroke, HF, and CVD death) were selected for model inclusion based on available probabilities and hazard ratios from published data on cardiovascular outcomes associated with TTR for systolic BP quartiles. ${ }^{8,9}$ Specifically, data on TTR for systolic $\mathrm{BP}$ quartiles and cardiovascular outcomes were derived from a post hoc analysis of the SPRINT trial, a randomized, controlled, open-label trial of intensive vs standard BP control. ${ }^{9}$

Patients within the SPRINT trial were censored after their first cardiovascular event, precluding analysis of subsequent events. Accordingly, cardiovascular events in this study were assumed to be mutually exclusive. Patients who did not incur 1 of these 4 events were assumed to have had no major cardiovascular event.

\section{HYPERTENSION MANAGEMENT AND CARDIOVASCULAR EVENT COSTS}

The model incorporated direct medical costs, including both programmatic costs (ie, direct costs for provider time) and downstream health care utilization associated 


\begin{tabular}{|c|c|c|c|}
\hline Variables & $\begin{array}{c}\text { Base-case } \\
\text { value }\end{array}$ & Range & Reference \\
\hline \multicolumn{4}{|l|}{ Programmatic costs } \\
\hline Annual PPCCM pharmacist visits, $\mathrm{n}$ & 6 & $4-12$ & Dixon et al, $2020^{13}$ \\
\hline PPCCM cost per visit, \$ & 24 & $19-29$ & ASHP, $2019^{21}$ \\
\hline \multicolumn{4}{|l|}{ Annual physician visits } \\
\hline PPCCM group, $\mathrm{n}$ & 1 & $1-2$ & Assumption \\
\hline Usual care visits, $\mathrm{n}$ & 3 & $1-6$ & Dixon et al, $2020^{13}$ \\
\hline Physician cost per visit, $\$$ & 90 & $72-108$ & CMS, 201922 \\
\hline Total cost of PPCCM, \$ & 702 & $562-842$ & ASHP, $2019^{21}$ \\
\hline Total cost of usual care, $\$$ & 810 & $648-972$ & CMS, $2019^{22}$ \\
\hline \multicolumn{4}{|l|}{ Downstream health care costs, \$ } \\
\hline One-time cost of nonfatal MI & 24,089 & $15,372-32,306$ & Bress et al, $2017^{19}$ \\
\hline One-time cost of stroke & 15,678 & $6,001-42,039$ & Bress et al, $2017^{19}$ \\
\hline One-time cost of heart failure & 11,678 & $11,669-16,580$ & Bress et al, $2017^{19}$ \\
\hline One-time cost of CVD death & 19,514 & $12,560-33,024$ & Bress et al, $2017^{19}$ \\
\hline \multicolumn{4}{|c|}{$\begin{array}{l}\text { ASHP=American Society for Health-Systems Pharmacists; } B P=\text { blood pressure; } C M S=\text { Centers for Medicare } \\
\text { \& Medicaid Services; } C V D=\text { cardiovascular disease; } M I=\text { myocardial infarction; } P P C C M=\text { pharmacist- } \\
\text { physician collaborative care model; } T T R=\text { time in target range. }\end{array}$} \\
\hline
\end{tabular}

with cardiovascular events (Table 1). Cost data were obtained from publicly available data and recently published cost-effectiveness analyses. ${ }^{19,21,22}$

Provider visit utilization data were obtained from a real-world analysis of PPCCM vs usual care for the management of hypertension. ${ }^{13}$ Specifically, for the cost of the PPCCM program, patients were assumed to have been seen for hypertension management 6 times per year by a pharmacist ${ }^{13}$ and once per year by a physician. Subjects in the usual care group were assumed to be seen 3 times per year by a physician. ${ }^{13}$

The cost per pharmacist visit reflected Current Procedural Terminology (CPT) code 99211 (level 1), an "incident-to" billing code used by pharmacists given a lack of provider status and eligibility to bill at a higher level. ${ }^{21}$ For usual care visits, the CPT code 99213 was used for evaluation and management/outpatient visits. ${ }^{22}$ performed varying the cost per pharmacist visit, the number of annual pharmacist visits among patients in the PPCCM program, and the number of annual physician visits among patients in usual care to assess the values at which the programmatic costs of the 2 models would be equal.

\section{Results}

In base-case analyses, PPCCM hypertension management was associated with lower total program costs (difference: $-\$ 108.00)$ and lower downstream medical expenditures (difference: -\$162.86) when compared to usual care (Table 2). For every 10,000 hypertension patients managed with PPCCM vs usual care over a 3-year time horizon, approximately 27 CVD deaths, 29 strokes, 21 nonfatal MIs, and 12 incident HF diagnoses are expected to be averted.

PPCCM was associated with lower downstream medical expenditures across all parameter ranges tested in the deterministic sensitivity analysis. The expected downstream health care savings were most sensitive to the likelihood that patients receiving usual care spend little to no time in therapeutic systolic BP range (TTR for systolic BP: 0\%-25\%; Figure 2). PPCCM was expected to reduce health care expenditures even as the proportion of usual care patients with TTR for systolic BP of $0 \%-25 \%$ was varied from its base-case value of $55 \%$, the probability observed by Dixon et al, ${ }^{13}$ to the lowest probability tested, $40 \%$.

The program costs of hypertension management with PPCCM, while lower than those of usual care in base-case analyses, were sensitive to the number of visits with a physician (usual care patients) and pharmacist (PPCCM patients; Figure 3). Due to the substantial difference in CPT code reimbursement for pharmacist vs usual care visits, a patient in 


\section{TABLE 2 Cost-Effectiveness Results}

\begin{tabular}{|c|c|c|c|}
\hline & PPCCM & Usual care & Difference \\
\hline \multicolumn{4}{|l|}{ Cardiovascular events } \\
\hline Nonfatal MI & 0.0300 & 0.0321 & 21 per 10,000 \\
\hline Stroke & 0.0149 & 0.0178 & 29 per 10,000 \\
\hline Heart failure & 0.0225 & 0.0237 & 12 per 10,000 \\
\hline CVD death & 0.0116 & 0.0143 & 27 per 10,000 \\
\hline Total downstream health care expenditures, \$ & $1,535.82$ & $1,698.64$ & $-\$ 162.82$ \\
\hline Total program costs, $\$$ & 702.00 & 810.00 & $-\$ 108.00$ \\
\hline Cost-benefit ratio & Dominant & & \\
\hline
\end{tabular}

$C V D=$ cardiovascular disease $: M I=$ myocardial infarction; $P P C C M=$ pharmacist - physician collaborative care model.

the PPCCM program who was seen 6 times per year by a pharmacist and once per year by a physician was still cheaper than a patient in the usual care group who was seen 3 times per year by a physician.

However, in 1-way sensitivity analysis, the cost of PPCCM hypertension management exceeded the cost of usual care when independently varying the number of both types of provider visits. First, if the number of hypertension-related physician visits each year was reduced from 3 to 1 while holding the number of PPCCMrelated visits constant, the cost of the PPCCM hypertension management exceeded the cost of usual care by $\$ 432$ over the 3-year study period. Second, when the number of pharmacist visits among patients enrolled in PPCCM increased from 6 per year to 12 while the number of physician visits in the usual care group $(n=3)$ was held constant, PPCCM was associated with an incremental program cost of $\$ 324$ over usual care.

In a threshold analysis, the costs of the PPCCM and usual care programs became equal when the unit cost of pharmacist visits were increased $62.5 \%$, to $\$ 39$. The program costs were also equal when the number of PPCCM patient visits increased from
6 to 10 pharmacist visits per year, or the number of usual care patient visits decreased from 3 to 2 physician visits per year.

\section{Discussion}

This study quantifies the cost-effectiveness of PPCCM for hypertension management to improve BP control and cardiovascular outcomes. Previous studies evaluated PPCCM impact on TTR for systolic $\mathrm{BP}^{13}$ and the association between TTR for systolic BP and cardiovascular outcomes, ${ }^{8}$ but no pharmacoeconomic analysis had combined these findings to model the cost-effectiveness of PPCCM from the payer perspective.

This study found that patients enrolled in the PPCCM incurred fewer costs associated with their direct hypertension management. The lower PPCCM program costs reflect the significantly lower cost of pharmacist time as billed by "incident to" hypertension.

In a threshold analysis, the direct cost of provider time was lower for usual care if patients receiving usual care had fewer than 2 physician visits per year. However, previous studies CPT codes than physician visits for suggest that approximately $80 \%$ of adult patients with hypertension have 2 or more hypertension-focused physician visits per year. ${ }^{24}$ Nonetheless, given that the cost of PPCCM hypertension management exceeded the cost of usual care among patients with only 1 hypertension-related physician visit each year, payers concerned with the immediate budget impact of PPCCM reimbursement may focus on coverage for patients with at least 2 or 3 hypertension-related physician visits annually, as PPCCM is cost-neutral and cost-saving, respectively, in these populations.

A second threshold analysis found that the direct program cost of PPCCM would equal that of usual care if patients met 10 times with a pharmacist annually. This well exceeds the number of previously observed pharmacist appointments for patients in 2 different PPCCM programs, ${ }^{13,25}$ suggesting that the PPCCM model is likely to save upfront hypertension management costs from the payer perspective.

While this study found that the direct intervention costs of the PPCCM were lower than those of usual care, several previous cost-effectiveness analyses on pharmacist-physician collaborative care for the management of hypertension found increased costs for patients in a PPCCM..$^{25,26}$ A cost-effectiveness analysis from a societal perspective on a physicianpharmacist collaboration to improve hypertension control conducted by Polgreen et al reported that provider costs over a 9-month period were \$238.96 for PPCCM patients and \$113.67 for usual care patients managed only by a physician. ${ }^{25}$ Rather than using CPT billing codes, that study determined costs based on time spent with pharmacists and providers and their average compensation rates, likely due to its societal, rather than 


\section{FIGURE 2 Tornado Diagram of Incremental Downstream Health Care Expenditures Among Patients Receiving PPCCM vs Usual Care}

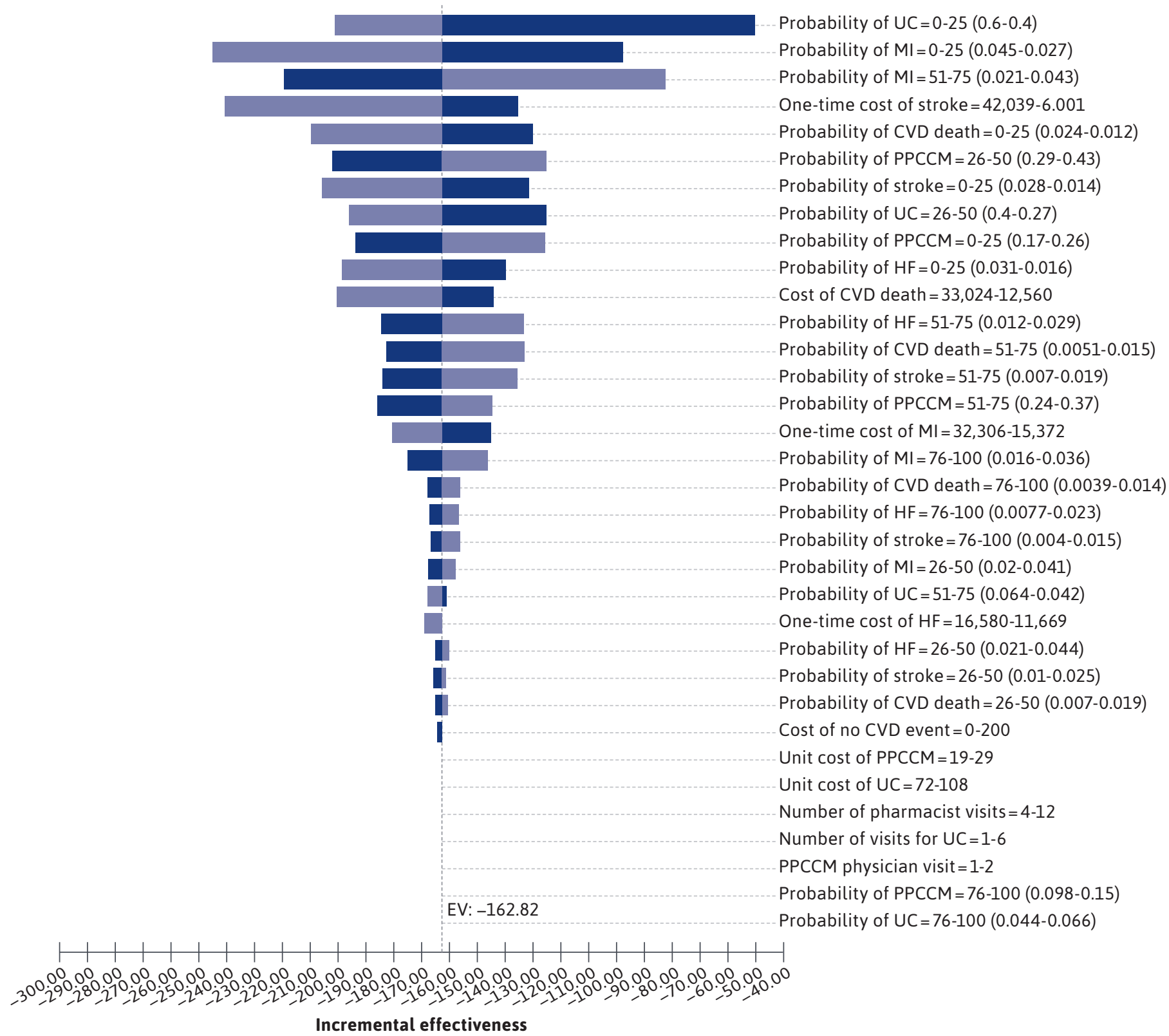

$C V D=$ cardiovascular disease; $E V=$ expected value; $H F=$ heart failure; $M I=$ mycardial infarction; $P P C C M=$ pharmacist-physician collaborative care model; $U C=$ usual care.

payer, perspective. Thus, while usual care patients had the same number of physician visits (median: 3 visits), as was assumed for this analysis, the cost of those 3 visits was calculated to be only $\$ 113.67$. Kulchaitanaroaj et al similarly reported higher costs for PPCCM in 2 analyses, ${ }^{26,27}$ but, like Polgreen, ${ }^{25}$ used time-based costing, resulting in higher provider costs among PPCCM patients $(\$ 345.25)$ than those in usual care $(\$ 111.84) .{ }^{27}$ 


\section{FIGURE 3 Tornado Diagram of Incremental Cost of PPCCM vs Usual Care}

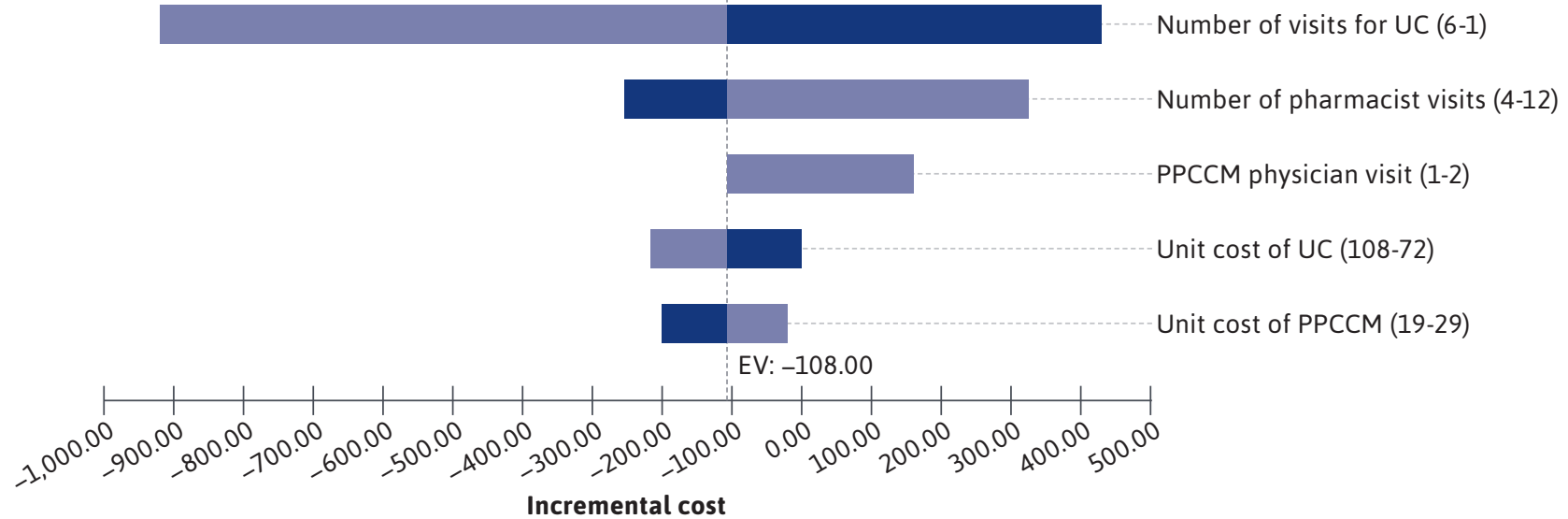

EV=expected value; $P P C C M=$ pharmacist physician collaborative care model; $U C=$ usual care .

The use of CPT codes in this analysis generated higher expected costs for physician visits but more accurately reflects hypertension management costs from the payer perspective. CPT "incident-to" billing, in which the physician bills, receives payment, and reimburses the pharmacist, offers payers an opportunity to implement payment for services within existing frameworks of physician reimbursement. The use of incident-to billing and collaborative practice agreements may also reduce barriers to PPCCM implementation from the pharmacy perspective, as a lack of clear reimbursement was cited by study authors as a potential barrier to more widespread PPCCM dissemination. Physician champions for the model can help to facilitate reimbursement efforts and streamline referrals, as the pharmacists practicing under the collaborative practice agreement in the collaborative care model routinely reported encountering new complaints from patients.

When compared with usual care, PPCCM was associated with lower downstream health care expenditures, saving an expected $\$ 162.82$ over a 3-year time horizon. Our finding of downstream health care savings is consistent with the majority of economic evaluations of clinical pharmacy services for chronic disease state management that incorporate long-term health care expenditures. ${ }^{28}$ Pharmacist-delivered medication management and hypertension education have consistently been shown to reduce $\mathrm{BP},{ }^{29}$ which, in turn, is associated with fewer cardiovascular events. Further, the more frequent pharmacist interactions in the PPCCM model may have facilitated the development of a stronger patient-pharmacist relationship and higher levels of trust, thereby enabling patients to better manage their chronic diseases. ${ }^{30}$

Our study reported that, for every 10,000 patients with hypertension managed in a PPCCM model, 27 CVD deaths, 29 strokes, 21 nonfatal MIs, and 12 incident HF diagnoses are expected to be averted. While quality-adjusted life-years (QALYs) were not utilized as an outcome in this study, past cost-utility analyses of pharmacist-led or collaborative hypertension management have reported such programs to be cost-effective. Bryant et al modeled 10-year health outcomes and 1-year health care costs associated with pharmacist-led hypertension care in Black-owned barbershops in the Los Angeles Barbershop Blood Pressure Study (LABBPS) from a health care sector perspective. ${ }^{14}$ They reported a mean cost of $\$ 42,717$ per QALY gained. Kulchaitanaroaj et al similarly reported a PPCCM to be highly cost-effective from the payer perspective $(\$ 26,807$ per QALY gained).

This study thus adds to a growing body of literature suggesting that pharmacist collaboration in the management of chronic conditions not only benefits the health outcomes of the patient but does so in a cost-effective manner. ${ }^{31-33}$ PPCCMs may have other benefits not captured in economic evaluations, including decreased physician workload and an ability to reach underserved populations. ${ }^{34}$

\section{FUTURE RESEARCH}

This research reports the cost-benefit of PPCCMs vs usual care on TTR for systolic BP for 4 cardiovascular outcomes. The data for TTR for systolic BP and cardiovascular outcomes 
was from previously published data. The direct effect of PPCCM and usual care as it relates to patient outcomes and costs has not been reported. Our study was designed to evaluate only the first occurrence of CVD or death. Therefore, future research that includes a Markov model investigating recurrent cardiovascular events should be conducted. Additionally, the cost-benefit of PPCCM with the addition of hypertensive medication costs should also be explored. Different cardiovascular outcomes can result in additional hypertensive medications, which can affect costs from the payer perspective.

\section{LIMITATIONS}

This research included several limitations. TTR for systolic BP data was collected from a study with a small population of 112 patients (56 patients in both PPCCM and usual care), which may limit generalizability ${ }_{1}^{13}$ though the impact of the PPCCM on hypertension management reported by Dixon et $\mathrm{al}^{13}$ was similar to that reported elsewhere. ${ }^{17,18}$

This study did not incorporate the cost of medications due to the lack of information on medication utilization among patients in the 2 groups. The post hoc analysis of SPRINT data by Fatani et al indicated the number of BP-lowering agents based on participants' TTR for systolic $\mathrm{BP},{ }^{8}$ but it is not known whether pharmacist involvement to promote higher TTR for systolic BP would systematically change the number of BP-lowering agents required to improve $\mathrm{BP}$ control.

While Dixon et al did not report specific medication utilization in the PPCCM and usual care groups, ${ }^{13}$ the antihypertensives used by both PPCCM and usual care patients were predominately low-cost generics, minimizing the effect of drug costs on the cost-effectiveness of the program. Additionally, indirect costs were not included in our analysis due to a lack of data linking TTR for systolic BP to changes in productivity, absenteeism, and other indirect costs; similarly, utility values have not yet been established by TTR for systolic BP ranges. However, given that numerous adverse cardiovascular outcomes have been associated with indirect costs of lost productivity due to morbidity and mortality, ${ }^{35}$ it is likely that the lack of indirect costs in this study resulted in an underestimation of the downstream savings associated with PPCCMs.

Future research is needed to assess indirect costs and potential changes in QALYs associated with improvements in TTR for systolic BP. Furthermore, costs for payer oversight, including quality assurance/auditing of the benefit, were not considered; substantial quality assurance costs may reduce the reported savings associated with PPCCM implementation.

This study evaluated the impact of hypertension management with PPCCM on cardiovascular outcomes and associated costs over a 3-year time frame. Hypertension is a chronic disease and is linked to health consequences including multiple MIs, strokes, and HF exacerbations. The data we utilized from the SPRINT trial censored patients after their first occurrence of a major adverse cardiovascular event during the study period. Therefore, our study may have underestimated the impact of PPCCM on long-term adverse cardiovascular events associated with TTR for systolic BP in hypertension management.

Finally, this study used effectiveness estimates from a real-world study on PPCCM for hypertension management..$^{13}$ However, a recent nationwide survey found that only about half of patients considered themselves likely to participate in clinical pharmacy services under a collaborative practice agreement, despite their perceptions that such services improve physicianpharmacist coordination. ${ }^{36}$ If eligible patients choose not to participate in PPCCM services where available, the scope of downstream benefits realized by widespread programmatic access would be more limited than with widespread adoption.

\section{Conclusions}

This is the first study to evaluate the cost-benefit of a PPCCM and usual care on TTR for systolic BP in patients with hypertension. The results indicated that a PPCCM was less costly to administer and resulted in reduced downstream adverse cardiovascular events and health care savings relative to usual care. Although further research is needed to evaluate the long-term costs and outcomes of PPCCM, payer coverage of PPCCM services may prevent future health care costs and improve patient cardiovascular outcomes.

\section{DISCLOSURES}

No funding was received for the completion of this research. The authors have nothing to disclose.

Study results were presented as an abstract at the AMCP 2021 Virtual, April 12-16, 2021.

\section{REFERENCES}

1. Centers for Disease Control and Prevention. Facts about hypertension. 2020. Accessed January 8, 2021. https:// www.cdc.gov/bloodpressure/facts.htm 
2. Whelton PK, Carey RM, Aronow WS, et al. 2017 ACC/AHA/AAPA/ABC/ACPM/ AGS/APhA/ASH/ASPC/NMA/PCNA guideline for the prevention, detection, evaluation, and management of high blood pressure in adults: a report of the American College of Cardiology/ American Heart Association task force on clinical practice guidelines. Hypertension. 2018;71:e13-e115. doi:10.1161/ HYP.0000000000000065

3. Kirkland EB, Heincelman M, Bishu KG, et al. Trends in healthcare expenditures among US adults with hypertension: national estimates, 2003-2014. J Am Heart Assoc. 2018;7(11):2003-14. doi:10.1161/ JAHA.118.008731

4. Gosmanova EO, Mikkelsen MK, Molnar MZ, et al. Association of systolic blood pressure variability with mortality, coronary heart disease, stroke, and renal disease. J Am Coll Cardiol. 2016;68(13):1375-86. doi:10.1016/j. jacc.2016.06.054

5. Wang J, Shi X, Ma C, et al. Visit-to-visit blood pressure variability is a risk factor for all-cause mortality and cardiovascular disease: a systematic review and metaanalysis. J Hypertens. 2017;35(1):10-17. doi:10.1097/HJH.0000000000001159

6. Clark D, Nicholls SJ, St John J, et al. Visit-to-visit blood pressure variability, coronary atheroma progression, and clinical outcomes. JAMA Cardiol. 2019;4(5):437-43. doi:10.1001/ jamacardio.2019.0751

7. Doumas M, Tsioufis C, Fletcher R, Amdur R, Faselis C, Papademetriou V. Time in therapeutic range, as a determinant of all-cause mortality in patients with hypertension. J Am Heart Assoc. 2017;6(11). doi:10.1161/JAHA.117.007131

8. Fatani N, Dixon DL, Van Tassell BW, Fanikos J, Buckley LF. Systolic blood pressure time in target range and cardiovascular outcomes in patients with hypertension. J Am Coll Cardiol. 2021;77(10):1290-99. doi:10.1016/j. jacc.2021.01.014
9. SPRINT Research Group. A randomized trial of intensive versus standard blood-pressure control. N Engl J Med. 2015;373(22):2103-16. doi:10.1056/ nejmoa1511939

10. Wagner EH. The role of patient care teams in chronic disease management. $\mathrm{Br}$ Med J. 2000;320(7234):569-72. doi:10.1136/ bmj.320.7234.569

11. Manolakis PG, Skelton JB. Pharmacists' contributions to primary care in the United States collaborating to address unmet patient care needs: the emerging role for pharmacists to address the shortage of primary care providers. Am J Pharm Educ. 2010;74(10). doi:10.5688/ aj7410S7

12. Isetts BJ, Buffington DE, Carter BL, Smith M, Polgreen LA, James PA. Evaluation of pharmacists' work in a physician-pharmacist collaborative model for the management of hypertension. Pharmacotherapy. 2016;36(4):374-84. doi:10.1002/phar.1727

13. Dixon DL, Parod ED, Sisson EM, Van Tassell BW, Nadpara PA, Dow A. Impact of a pharmacist-physician collaborative care model on time-in-therapeutic blood pressure range in patients with hypertension. J Am Coll Clin Pharm. 2020;3(2):404-09. doi:10.1002/jac5.1115

14. Bryant K, Moran A, Kazi D, et al. Costeffectiveness of hypertension treatment by pharmacists in Black barbershops. Circulation. Published online 2021. doi:10.1161/CIRCULATIONAHA.120.051683

15. Schoenthaler AM, Lancaster KJ, Chaplin W, Butler M, Forsyth J, Ogedegbe G. Cluster randomized clinical trial of FAITH (Faith-Based Approaches in the Treatment of Hypertension) in Blacks. Circ Cardiovasc Qual Outcomes. 2018;11(10):e004691. doi:10.1161/ CIRCOUTCOMES.118.004691

16. Matzke GR, Moczygemba LR, Williams KJ, Czar MJ, Lee WT. Impact of a pharmacist-physician collaborative care model on patient outcomes and health services utilization. Am J Health Syst Pharm. 2018;75(14):1039-47. doi:10.2146/ ajhp170789
17. Carter BL, Clarke W, Ardery G, et al. A cluster-randomized effectiveness trial of a physician-pharmacist collaborative model to improve blood pressure control. Circ Cardiovasc Qual Outcomes. 2010;3(4): 418-23. doi:10.1161/CIRCOUTCOMES. 109.908038

18. Carter BL, Ardery G, Dawson JD, et al. Physician/pharmacist collaboration to improve blood pressure control. Arch Intern Med. 2010;169(21):1996-2002. doi:10.1001/archinternmed.2009.358. Physician/Pharmacist

19. Bress AP, Bellows BK, King JB, et al. Cost-effectiveness of intensive versus standard blood-pressure control. N Engl J Med. 2017;377(8):745-55. doi:10.1056/ NEJMsa1616035.Cost-Effectiveness

20. Kim DD, Wilkinson C, Kim DD, et al. The influence of time horizon on results of cost-effectiveness analyses. Expert Rev Pharmacoecon Outcomes Res. doi:10.1080/ 14737167.2017.1331432

21. American Society of Health-System Pharmacists. Pharmacist billing/coding quick reference sheet for services provided in physician-based clinics. June 2019. Accessed April 1, 2021. https://www.ashp.org/-/media/assets/ pharmacy-practice/resource-centers/ ambulatory-care/billing-quick-referencesheet.pdf

22. Centers for Medicare \& Medicaid Services. CY 2019 Medicare Physician Fee Schedule final rule summary. Accessed April 1, 2021. https://www.cms.gov/Medicare/ Medicare-Fee-for-Service-Payment/ PhysicianFeeSched

23. Nguyen Q, Dominguez J, Nguyen L, Gullapalli N. Hypertension management: an update. Am Health Drug Benefit. 2010;3(1):47-55.

24. Ashman JJ, Rui P, Schappert SM. Age differences in visits to office-based physicians by adults with hypertension: United States, 2013. Natl Cent Health Stat. 2016;(263):1-8. 
25. Polgreen LA, Han J, Carter BL, et al. Cost-effectiveness of a physician-pharmacist collaboration intervention to improve blood pressure control. Hypertension. 2015;66(6):1145-51. doi:10.1161/

HYPERTENSIONAHA.115.06023

26. Kulchaitanaroaj P, Brooks JM, Chaiyakunapruk N, Goedken AM, Chrischilles EA, Carter BL. Cost-utility analysis of physician-pharmacist collaborative intervention for treating hypertension compared with usual care. J Hypertens. 2017;35(1):178-87. doi:10.1097/ HJH.0000000000001126

27. Kulchaitanaroaj P, Brooks JM, Ardery G, Newman D, Carter BL. Incremental costs associated with physician and pharmacist collaboration to improve blood pressure control. Pharmacotherapy. 2012;32(8):772-80. doi:10.1002/j.1875-9114.2012.01103.x

28. Talon B, Perez A, Yan C, et al. Economic evaluations of clinical pharmacy services in the United States: 2011-2017. J Am Coll Clin Pharm. 2020;3(4):793-806. doi:10.1002/jac5.1199
29. Omboni S, Tenti M, Coronetti C. Physician-pharmacist collaborative practice and telehealth may transform hypertension management. J Hum Hypertens. 2019;33:177-87. doi:10.1038/ s41371-018-0147-x

30. Ayub M, Gul S, Afzal N, Eraj A. An effective clinical pharmacist communication may built patient trust on pharmacist over other healthcare providers: evidence based practice. Int J Pharm Sci Res. 2015;6(10):4442-47. doi:10.13040/ IJPSR.0975-8232.6(10).4442-47

31. Houle SKD, Chuck AW, McAlister FA, Tsuyuki RT. Effect of a pharmacist-managed hypertension program on health system costs: an evaluation of the study of cardiovascular risk intervention by pharmacists-hypertension (SCRIP-HTN). Pharmacotherapy. 2012;32(6):527-37. doi:10.1002/j.1875-9114.2012.01097.x

32. Wang Y, Yeo QQ, Ko Y. Economic evaluations of pharmacist-managed services in people with diabetes mellitus: a systematic review. Diabet Med. 2016;33(4):421-27. doi:10.1111/dme.12976
33. Snider M, Carnes C, Grover J, Davis R, Kalbfleisch S. Cost-benefit and cost-savings analyses of antiarrhythmic medication monitoring. Am J Health Pharm. 2012;69(18):1569-73. doi:10.2146/ ajhp110270

34. Hwang AY, Gums TH, Gums JG, Uni HP. The benefits of physicianpharmacist collaboration. J Fam Pract. 2017;66(12):e1-e8.

35. Elliott WJ. The economic impact of hypertension. J Clin Hypertens (Greenwich). 2003;5(3 Suppl 2):3-13. doi:10.1111/j.1524-6175.2003.02463.x

36. Schumacher L, Coe AB, Lester CA, Rothermal M, Dorsch MP, Dorsch MP. Factors that influence patient acceptance of clinical pharmacy services: a nationwide survey. J Am Pharm Assoc. 2020;60:1058-1067. doi:10.1016/j. japh.2020.08.042 